\title{
Histone deacetylase inhibition activates Nrf2 and protects against osteoarthritis
}

\author{
Dawei Cai ${ }^{1,2}$, Shasha Yin ${ }^{1}$, Jun Yang ${ }^{1}$, Qing Jiang ${ }^{1,2,3^{*}}$ and Wangsen Cao ${ }^{1,4^{*}}$
}

\begin{abstract}
Introduction: Osteoarthritis $(\mathrm{OA})$ is a common joint disease that can cause gradual disability among the aging population. Nuclear factor (erythroid-derived 2)-like 2 (Nrf2) is a key transcription factor that regulates the expression of phase II antioxidant enzymes that provide protection against oxidative stress and tissue damage. The use of histone deacetylase inhibitors (HDACi) has emerged as a potential therapeutic strategy for various diseases. They have displayed chondroprotective effects in various animal models of arthritis. Previous studies have established that Nrf2 acetylation enhances Nrf2 functions. Here we explore the role of Nrf2 in the development of OA and the involvement of Nrf2 acetylation in HDACi protection of OA.

Methods: Two OA models - monosodium iodoacetate (MIA) articular injection and destabilization of the medial meniscus (DMM) - were used with wild-type (WT) and Nrf2-knockout (Nrf2-KO) mice to demonstrate the role of Nrf2 in OA progression. A pan-HDACi, trichostatin A (TSA), was administered to examine the effectiveness of $\mathrm{HDACi}$ on protection from cartilage damage. The histological sections were scored. The expression of OA-associated matrix metalloproteinases (MMPs) 1, 3, and 13 and proinflammatory cytokines tumor necrosis factor (TNF)-a, interleukin (IL)-1 1 , and IL-6 were assayed. The effectiveness of HDACi on OA protection was compared between WT and Nrf2-KO mice.

Results: Nrf2-KO mice displayed more severe cartilage damage in both the MIA and DMM models. TSA promoted the induction of Nrf2 downstream proteins in SW1353 chondrosarcoma cells and in mouse joint tissues. TSA also reduced the expression of OA-associated proteins MMP1, MMP3, and MMP13 and proinflammatory cytokines TNF- $\alpha$, IL-1 $\beta$, and IL-6. TSA markedly reduced the cartilage damage in both OA models but offered no significant protection in Nrf2-KO mice.
\end{abstract}

Conclusions: Nrf2 has a major chondroprotective role in progression of OA and is a critical molecule in HDACi-mediated OA protection.

\section{Introduction}

Osteoarthritis (OA) is a common joint disease and the major cause of disability among the aging population. OA is characterized by progressive degradation in articular cartilage, periarticular bone, synovial joint lining, and adjacent supporting connective tissue elements, which eventually results in a loss of joint function [1]. Although many etiological factors contribute to OA disease progression, such as hereditary, metabolism, and mechanical stress $[2,3]$, the exact mechanism of OA remains unclear. Currently, there are no satisfactory drugs for effective treatment of OA, and total joint replacement has to be considered in severe cases.

\footnotetext{
*Correspondence: qingj@nju.edu.cn; wangsencao@nju.edu.cn

1Jiangsu Key Laboratory of Molecular Medicine, Nanjing University School of Medicine, Nanjing 210093, People's Republic of China

Full list of author information is available at the end of the article
}

Nuclear factor (erythroid-derived 2)-like 2 (Nrf2) is a key transcription factor that regulates the antioxidant defense system. Nrf2 activates its downstream gene expression by controlling the antioxidant response elements (AREs) located in the promoter regions of its target genes, including antioxidative enzyme heme oxygenase $1(\mathrm{HO}-1)$ and NAD(P)H:quinine oxidoreductase 1 (NQO1) [4]. Nrf2 activity is regulated by various protein modification processes, such as Keap1-mediated ubiquitinated degradation, protein kinase $\mathrm{C} /$ mitogen-activated protein kinase (MAPK)-mediated phosphorylation $[5,6]$, and histone acetyltransferase (HAT)/histone deacetylase (HDAC)-mediated acetylation [7]. Nrf2 acetylation enhances its transcription capacity and downstream target expression and has been shown to confer protection in animal models of inflammation- and oxidative stress-related disease $[7,8]$. 
HDACs can alter the acetylation status of histone and non-histone proteins and can regulate many physiological and pathological processes. Histone deacetylase inhibitors $(\mathrm{HDACi})$ have therapeutic potential in various diseases [9-11]. Inhibition of HDACs causes hyperacetylation of the target proteins and leads to an alteration of gene expression involved in cell differentiation, proliferation, or apoptosis [12]. Mounting evidence demonstrates that HDACi prevent degradation of cartilage in animal models of OA [13-16], suggesting that HDACs have a protective role in OA. However, the molecular mechanisms underlying the action of HDACi in OA have not been fully elucidated.

Because Nrf2 and its downstream proteins are protective in OA-related joint damage and Nrf2 acetylation enhances Nrf2 functions, we hypothesize that Nrf2 acetylation plays an essential role in the protective effects of HDACi in OA. In this study, we explored the role of Nrf2 in the development of OA and the involvement of Nrf2 in the protective effects of HDACi in OA. We used two OA mouse models-monosodium iodoacetate (MIA) articular injection and destabilization of the medial meniscus (DMM) - to test the role of Nrf2 in the progression of OA. We further determined the requirement of Nrf2 in HDACi protection from OA in both MIA and DMM mice. Our results demonstrate that Nrf2 plays a major chondroprotective role in the progression of $\mathrm{OA}$ and is a critical mediator in $\mathrm{HDACi}$ protection from OA damage.

\section{Methods \\ Reagents}

Trichostatin A (TSA), MIA, and mouse recombinant interleukin (IL)-1 $\beta$ were obtained from Sigma-Aldrich (St. Louis, MO, USA). Anti-matrix metalloproteinase (antiMMP)-13, anti-MMP-3, anti-MMP-1, and anti-histone 3 antibodies were obtained from Santa Cruz Biotechnology (Santa Cruz, CA, USA). Anti-HO-1, anti-NQO1, and antiNrf2 antibodies were purchased from Bioworld Technology (Nanjing, China). Antiacetylated Nrf2 (K599) was obtained from ImmunoWay Biotechnology (Newark, DE, USA), and antiacetylated histone $\mathrm{H} 3$ was purchased from EMD Millipore (Billerica, MA, USA).

\section{Animals}

Nrf2-KO male mice (used with permission from Dr. Yamamoto Masayuki) and their wild-type (WT) littermates at 8-10 weeks of age were used in the experiments. Mice were fed a regular sterile chow diet and allowed access to water ad libitum, and they were housed under controlled conditions ( $25 \pm 2{ }^{\circ} \mathrm{C}$; 12 -h light-dark cycle). All experimental protocols were approved by the Institutional Animal Care and Use Committee (IACUC) of Nanjing University Medical School and were carried out in accordance with Nanjing University Medical School IACUC guidelines.
Genotypes of WT and Nrf2-KO mice were confirmed by polymerase chain reaction (PCR) of genomic DNA isolated from mouse tail (Fig. 1g). PCR amplification was carried out by using one common forward primer, a WT-specific reverse primer, and an $\mathrm{Nrf} 2-\mathrm{KO}-$ specific reverse primer in one PCR. The primer sequences are listed in Table 1.

\section{Models of osteoarthritis MIA model}

The MIA model of OA was adapted from a previous study [17]. Briefly, the mouse right knee joint was flexed at a 90-degree angle, and $6 \mu \mathrm{l}$ of $5 \mathrm{mg} / \mathrm{ml} \mathrm{MIA} \mathrm{dissolved}$ in sterile saline $(0.9 \%)$ was injected into the joint capsule with a 30-gauge needle. Intra-articular injection of saline into the left knee was performed as a control. Three weeks later, the mice were killed for joint and other analysis.

\section{DMM model}

The experimental mice were anesthetized with peritoneal injection of ketamine (50 $\mathrm{mg} / \mathrm{kg}$ ), and DMM surgery was performed with a microsurgical knife on the right knee by sectioning of the medial meniscotibial ligament (MMTL) that anchored the medial meniscus (MM) to the tibial plateau. The lateral meniscotibial ligament was identified and protected during the surgery. Contralateral knee joints received sham surgery in which the MMTL was exposed without sectioning. Mice were allowed completely free movement following DMM surgery. Six weeks later, the mice were killed and their knee joints were harvested for histological analysis and other examinations.

\section{Cell culture}

SW1353 human chondrosarcoma cells were grown in Dulbecco's modified Eagle's medium supplemented with $10 \%$ fetal bovine serum and $100 \mathrm{U} / \mathrm{ml}$ penicillin. Cells were maintained at $37{ }^{\circ} \mathrm{C}$ in a humidified incubator under a $5 \% \mathrm{CO}_{2}$ atmosphere. Cells were treated with different doses of TSA or in combination with IL-1 $\beta$ and then further examined.

\section{Treatment of TSA}

TSA was administrated by subcutaneous injection daily $(2.0 \mathrm{mg} / \mathrm{kg})$ for 3 weeks in the MIA model and for 6 weeks in the DMM model. TSA was dissolved in alcohol initially and then diluted with phosphate-buffered saline (PBS) to a final working concentration of $0.1 \mu \mathrm{g} / \mu \mathrm{l}$ before use $[9,10]$. Control mice received the same amount of PBS injection. At the end of the experiment, the mice were killed with $\mathrm{CO}_{2}$ inhalation and their knee joints were collected and stored at $-80{ }^{\circ} \mathrm{C}$ until further analysis. 


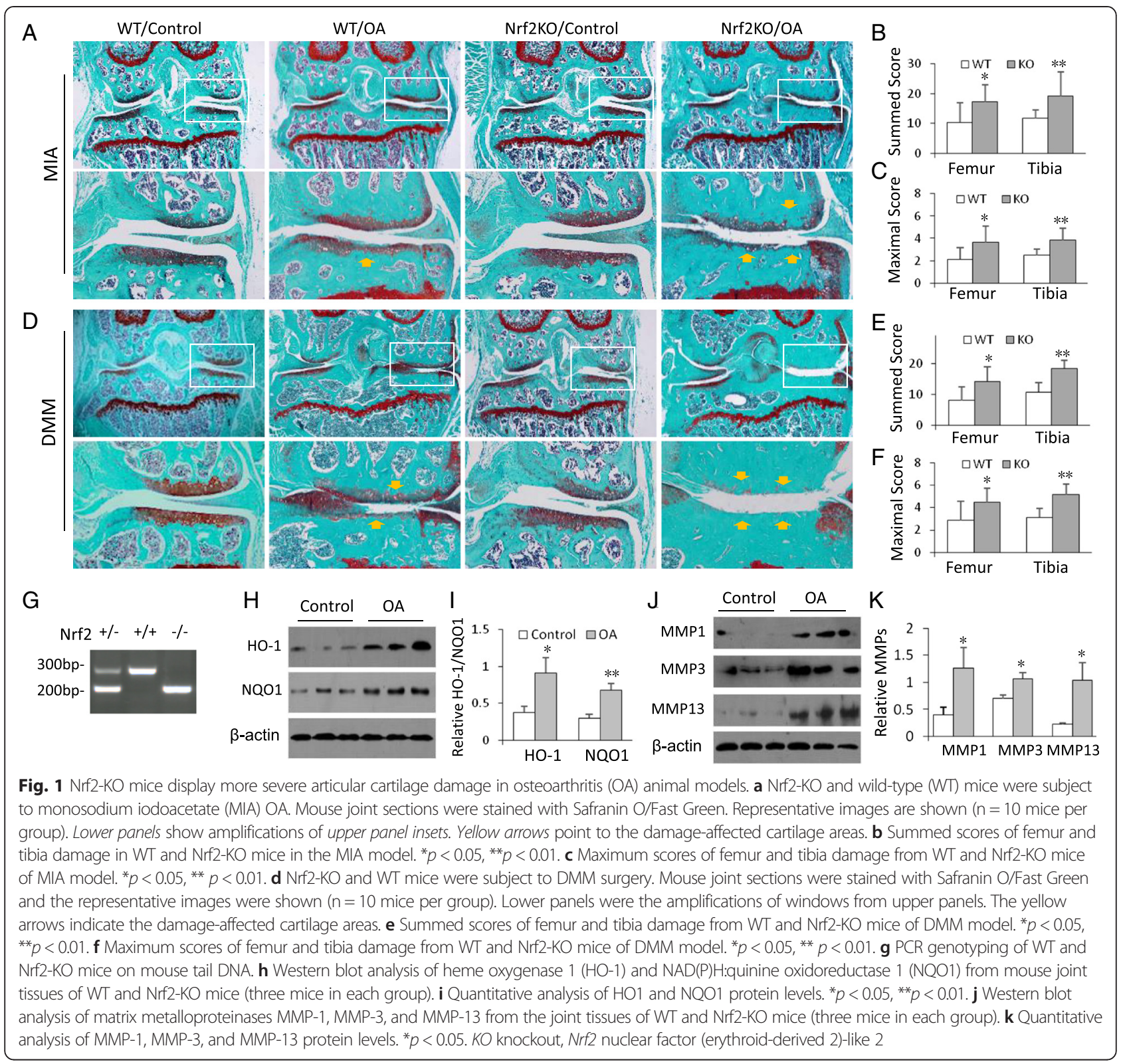

\section{Histological assessment}

Mouse knee joints were prepared for histological assessment. Briefly, the knee joints were prepared by removing skin and muscle and then immediately fixed in $4 \%$ paraformaldehyde. The knee joints were further decalcified in $20 \%$ formic acid and embedded in paraffin. Frontal serial sections ( $5 \mu \mathrm{m}$ thick) across entire joints were obtained, and 10 slides per joint at every $50 \mu \mathrm{m}$ were selected and stained with Safranin O/Fast Green to assess cartilage destruction.

The Osteoarthritis Research Society International (OARSI) scoring system (grade 0: surface intact, cartilage intact; grade 1: surface intact; grade 2: surface discontinuity; grade 3: vertical fissures; grade 4: erosion; grade
5: denudation; grade 6: deformation) was used to assess the joint cartilage degeneration [18]. The maximum score (the highest score in either quadrant of femur or tibia) and the summed score (the sum of the highest scores in all four quadrants of knee joint: medial femoral condyle, lateral femoral condyle, medial tibial plateau, and lateral tibial plateau) were used to measure the extent of cartilage destruction $[18,19]$.

\section{RT-PCR and qRT-PCR}

Total RNA was extracted from cultured chondrocytes using TRIzol reagent (Invitrogen, Carlsbad, CA, USA). cDNA was synthesized from total RNA using a reverse transcriptase cDNA synthesis kit (Vazyme Biotech, Piscataway, NJ, 
Table 1 RT-PCR and qRT-PCR primers

\begin{tabular}{|c|c|}
\hline Genes & RT-PCR primers \\
\hline MMP-1 & Forward: GTCAGGGGAGATCATCGG \\
\hline MMP-1 & Reverse: GCCCAGTACTTATTCCCT \\
\hline MMP-3 & Forward: ATGCCCACTTTGATGATGATGAAC \\
\hline MMP-3 & Reverse: CCACGCCTGAAGGAAGAGATG \\
\hline MMP-13 & Forward: GCTTAGAGGTGACTGGCAA \\
\hline MMP-13 & Reverse: CCGGTGTAGGTGTAGATAGGAA \\
\hline GAPDH & Forward: CTGCCGTCTAGAAAAACC \\
\hline GAPDH & Reverse: CCAAATTCGTTGTCATACC \\
\hline Nrf2GT & Forward: TGGACGGGACTATTGAAGGCTG \\
\hline Nrf2-WT & Reverse: CGCCTTITCAGTAGATGGAGG \\
\hline Nrf2-KO & Reverse: GCGGATTGACCGTAATGGGATAGG \\
\hline Genes & qRT-PCR primers \\
\hline $\mid L-1 \beta$ & Forward: ATGGCAGAAGTACCTAAGCTCGC \\
\hline IL-1 $\beta$ & Reverse: ACACAAATTGCATGGTGAAGTCAGTT \\
\hline TNF-a & Forward: ATGAGCACAGAAAGCATGATCCGC \\
\hline TNF-a & Reverse: CCAAAGTAGACCTGCCCGGACTC \\
\hline IL-6 & Forward: ACACACTGGTTCTGAGGGAC \\
\hline IL-6 & Reverse: TACCACAAGGTTGGCAGGTG \\
\hline MMP-1 & Forward: GCCACAAAGTTGATGCAGTT \\
\hline MMP-1 & Reverse: GCAGTTGAACCAGCTATTAG \\
\hline MMP-3 & Forward: ATGAAAATGAAGGGTCTTCCGG \\
\hline MMP-3 & Reverse: GCAGAAGCTCCATACCAGCA \\
\hline MMP-13 & Forward: ATGCATTCAGCTATCCTGGCCA \\
\hline MMP-13 & Reverse: AAGATTGCATTTCTCGGAGCCTG \\
\hline NQO1 & Forward: TITAGGGTCGTCTTGGCA \\
\hline NQO1 & Reverse: GTCTTCTCTGAATGGGCCAG \\
\hline $\mathrm{HO}-1$ & Forward: ACATCGACAGCCCCACCAAGTTCAA \\
\hline $\mathrm{HO}-1$ & Reverse: CTGACGAAGTGACGCCATCTGTGAG \\
\hline$\beta$-actin & Forward: TGACGGGGTCACCCACACTGTGCCCATCTA \\
\hline$\beta$-actin & Reverse: CTAGAAGCATTTGCGGTGGACGATGGAGGG \\
\hline \multicolumn{2}{|c|}{$\begin{array}{l}\text { GAPDH glyceraldehyde 3-phosphate dehydrogenase, } H O-1 \text { heme oxygenase } \\
1, I L \text { interleukin, } M M P \text { matrix metalloproteinase, NQO1 NAD(P)H:quinine } \\
\text { oxidoreductase 1, Nrf2 nuclear factor (erythroid-derived 2)-like 2; Nrf2-KO } \\
\text { Nrf2-knockout, } q R T-P C R \text { quantitative real-time polymerase chain reaction, } \\
R T-P C R \text { reverse transcription polymerase chain reaction, } T N F \text { tumor necrosis } \\
\text { factor, } W T \text { wild type }\end{array}$} \\
\hline
\end{tabular}

USA) according to the manufacturer's recommendations. The cDNA was amplified by PCR using specific primers and Taq DNA polymerase (Vazyme Biotech). PCR was preceded for 30-35 cycles in a TC-412 thermocycler (Techne/ Bibby Scientific, Stone, UK).

Quantitative real-time PCR (qRT-PCR) was performed with cells and joint tissues. Total RNA was extracted from mouse knee cartilage following homogenization in TRIzol reagent. Reverse transcription was performed with $1 \mu \mathrm{g}$ of RNA in a total 20- $\mu$ l reaction using PrimeScript RT Master Mix (Clontech Laboratories, Mountain View, CA,
USA) according to the manufacturer's instructions. qRTPCR experiments were done with a 7500 real-time PCR system (Applied Biosystems, Waltham, MA, USA) using SYBR Premix Ex Taq reagents (TaKaRa, Shiba, Japan). All data were normalized to $\beta$-actin. All assays were done in triplicate. The specific primer sets for qRT-PCR are listed in Table 1.

\section{Cartilage extraction and Western blot analysis}

Cartilage tissue was removed surgically and pulverized in liquid nitrogen shortly thereafter, then stored at $-80{ }^{\circ} \mathrm{C}$ until analysis. For Western blot analysis, the cartilage tissue was extracted in PBS containing $1 \%$ Triton X-100, $0.1 \%$ SDS, $20 \mathrm{nM}$ sodium orthovanadate, $1 \mu \mathrm{g} / \mathrm{ml}$ aprotinin, $1 \mathrm{mM}$ phenylmethylsulfonyl fluoride, and $5 \mathrm{mM}$ ethylenediaminetetraacetic acid. The homogenates were centrifuged at $12,000 \times g$ for 30 minutes at $4{ }^{\circ} \mathrm{C}$, and the protein concentration in the supernatant was measured using a bicinchoninic acid assay kit (Pierce Biotechnology/ Thermo Scientific, Rockford, IL, USA), Equal amounts of protein from each animal cartilage were solubilized in lysis buffer containing $10 \mathrm{mM}$ Tris- $\mathrm{HCl}$ (pH 7.5), $1 \mathrm{mM}$ ethylene glycol tetraacetic acid, $1 \mathrm{mM} \mathrm{MgCl} 2,1 \mathrm{mM}$ sodium orthovanadate, $1 \mathrm{mM}$ dithiothreitol, $0.1 \%$ mercaptoethanol, $0.5 \%$ Triton X-100, and protease inhibitor cocktail. Proteins were separated by SDS-PAGE and transferred onto a polyvinylidene difluoride membrane. The membranes were blocked with $5 \%$ milk resolved in Trisbuffered saline-Tween 20 buffer, followed by Western blotting with antibodies at predetermined dilutions.

\section{Luciferase assays}

An HO-1 promoter-luciferase reporter plasmid [20] was used to test Nrf2 signaling activity. HEK293 cells were cotransfected with the reporter plasmid and a Renilla luciferase reporter plasmid by using Lipofectamine 2000 reagent (Invitrogen). On the next day, the cells were treated with TSA for $12 \mathrm{~h}$, and a luciferase assay system (Promega, Madison, WI, USA) was used to measure luciferase activity. The relative luciferase activity in each sample was determined by subtracting the background firefly luciferase activity and normalized to Renilla luciferase activity. All reported assays were repeated at least three times independently.

\section{Statistical analysis}

Data were expressed as mean \pm standard deviation. Differences between groups were compared by Student's $t$ test or Mann-Whitney $U$ test. Analyses were performed using GraphPad Prism 4.0 software (GraphPad Software, La Jolla, CA, USA). A $p$ value $<0.05$ was considered statistically significant. A $p$ value $<0.01$ was considered statistically very significant. 


\section{Results \\ Nrf2-KO mice display accelerated progression of articular cartilage destruction in MIA and DMM models}

To investigate whether Nrf2 mediates HDACi protection against $\mathrm{OA}$, we first tested the role of $\mathrm{Nrf} 2$ in the development of OA in the MIA model. WT and Nrf2-KO mice were genotyped by PCR (Fig. 1g). Both WT and Nrf2-KO mice (10 mice in each group) received joint injections of MIA for 3 weeks before being killed. The joint sections were stained with Safranin O/Fast Green. Both WT and Nrf2-KO mice developed arthritis characterized by loss of joint cartilage and exposed subchondral bone with dense sclerosis. Compared with WT mice, Nrf2-KO mice showed much severer cartilage damage (Fig. 1a). To quantify the severity of the joint damage, we analyzed histopathological changes using the OARSI scoring system. WT mice displayed summed arthritic scores of $10.40 \pm 6.58$ for the femur and $11.75 \pm$ 2.79 for the tibia. The summed scores of Nrf2-KO mice were significantly higher at $17.25 \pm 5.76$ for the femur and $19.20 \pm 8.21$ for the tibia $(* p<0.05, * *<0.01)$ (Fig. 1b). WT mice had maximum scores of $2.15 \pm 1.00$ for the femur and $2.50 \pm 0.55$ for the tibia. The maximum scores of Nrf2-KO mice were also significantly higher (3.65 \pm 1.45 for the femur and $3.85 \pm 1.05$ for the tibia; ${ }^{*} p<0.05$, $* * p<0.01)$ (Fig 1c)

To confirm the importance of Nrf2 in the development of OA, we used another model of OA: a DMM model. We performed DMM surgery in the right knee joints of WT and Nrf2-KO mice (10 mice in each group). Six weeks later, the mouse knee joints were subject to histological analysis and the cartilage damage caused by instability was observed in the medial compartment when WT and Nrf2-KO mice were compared (Fig. 1d). WT mice displayed summed arthritic scores of $8.00 \pm 4.45$ for the femur and $10.65 \pm 3.04$ for the tibia. In contrast, Nrf2-KO mice showed significantly higher summed scores, with $14.15 \pm 4.81$ for the femur and $18.35 \pm 2.56$ for the tibia ( ${ }^{*} p<0.05$, ** $p<0.01$ ) (Fig. 1e). WT mice had maximum scores of $2.85 \pm 1.70$ for the femur and $3.15 \pm 0.74$ for the tibia. The maximum scores of Nrf2-KO mice were also significantly higher (femur $4.45 \pm 1.25$, tibia $5.15 \pm 0.98 ; \quad * p<0.05, \quad * *<0.01)$ (Fig. 1f).

We next examined the expression of two Nrf2 downstream proteins: HO-1 and NQO1. The expression of HO-1 and NQO1 was enhanced in OA cartilage (Fig. 1h, i), indicating that the Nrf2 signaling pathway was activated in OA. In addition, the expression of MMP-1, MMP-3, and MMP-13 was also increased in $\mathrm{OA}$ cartilage compared with the control cartilage (Fig. 1j, k). Taken together, these results indicate that $\mathrm{Nrf} 2$ is chondroprotective during the development of OA.

\section{HDAC inhibition activates Nrf2 signaling pathway in chondrocytes in vitro}

To test whether HDACi regulate Nrf2 signaling in chondrocytes, we examined the effect of TSA on the expression of Nrf2 downstream proteins in human chondrosarcoma SW1353 cells. The results showed that TSA induced the expression of the Nrf2 downstream proteins $\mathrm{HO}-1$ and NQO1 in a dose- and time-dependent manner (Fig. 2a-c). TSA also induced marked histone 3 acetylation, as expected (Fig. 2a, lower panel). TSA treatment caused Nrf2 nuclear translocation (Fig. 2d, e) and activated the $\mathrm{HO}-1$ promoter transactivation activity (Fig. 2f). To examine the effect of HDACi on OA-related protein expression, we used IL-1 $\beta$ to induce MMP expression in SW1353 cells. The results showed that TSA treatment suppressed IL-1 $\beta$-induced MMP-1, MMP-3, and MMP-13 expression at both the mRNA and protein levels (Fig. $2 g-i$ ), indicating that HDACi could effectively activate Nrf2 signaling and suppress OA-related MMP expression in chondrocytes.

\section{TSA alleviates OA-related cartilage damage}

To determine the protective property of HDACi on OA, we first tested the effect of TSA in the MIA model. TSA treatment alone did not cause an observable arthritis-like change to mouse knees (Fig. 3a). We injected MIA into mouse knees in the presence or absence of TSA $(2 \mathrm{mg} / \mathrm{kg} /$ day subcutaneously) for a period of 3 weeks. We then examined the knee joint histology by Safranin O/Fast Green staining. TSA significantly decreased the cartilage damage in the medial tibial plateau and femoral condyle (Fig. 3a, arrowheads). To better observe the tide mark and chondrocyte proliferation (cell clusters), we also performed hematoxylin and eosin staining, which clearly showed that the location of the tide mark in the MIA group was much closer to the articular surface, especially in the lateral femoral condyle of both femur and tibia, whereas the TSA-treated group displayed a much improved tide mark location and cell clusters (data not shown). Overall, the MIA group had summed scores of $10.33 \pm 3.59$ for the femur and $12.25 \pm 3.84$ for the tibia, whereas the TSA-treated group exhibited significantly lower values of $4.83 \pm 3.13$ for the femur and $6.17 \pm 3.27$ for the tibia $(p<0.05)$ (Fig. 3b). When the maximum score analysis was applied, TSA treatment demonstrated similar improvements. The MIA group had maximum scores of $2.08 \pm 0.67$ for the femur and $2.42 \pm 0.53$ for the tibia, whereas the TSA-treated group had significantly lower values of $0.92 \pm 0.67$ for the femur and $1.33 \pm 0.69$ for the tibia $(p<0.05)$ (Fig. 3c).

We also performed the experiments in the DMM model, TSA ( $2 \mathrm{mg} / \mathrm{kg} /$ day subcutaneously) was delivered for 6 weeks following surgical sectioning of the MMTL. TSA markedly relieved the cartilage damage (Fig. 3d). The DMM group had summed scores of $7.33 \pm 3.35$ for 


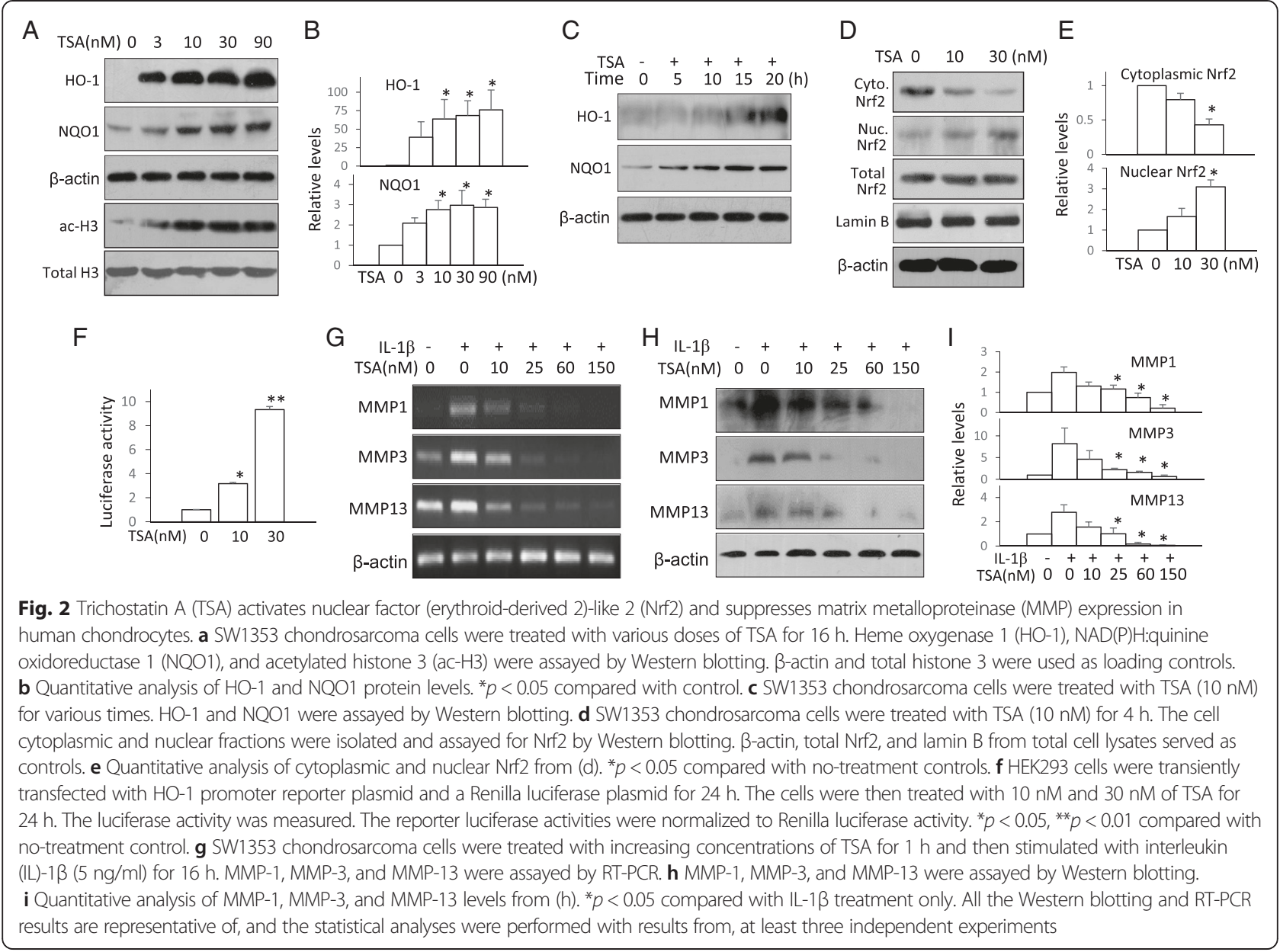

the femur and $8.17 \pm 2.73$ for the tibia, whereas the TSA-treated group exhibited significantly lower values of $2.00 \pm 1.26$ for the femur and $2.92 \pm 0.84$ for the tibia $(p<0.05)$ (Fig. 3e). The DMM group had maximum scores of $1.92 \pm 0.84$ for the femur and $2.5 \pm 1.35$ for the tibia, whereas the TSA-treated group had significantly lower values of $0.75 \pm 0.48$ for the femur and $1.17 \pm 0.24$ for the tibia $(p<0.05)$ (Fig. 3f). These results indicate that TSA prevention of cartilage damage was not modeldependent.

\section{TSA improves OA-associated protein expression}

To estimate the involvement of the Nrf2 pathway in TSA protection of $\mathrm{OA}$, we assayed $\mathrm{Nrf} 2$ downstream genes HO- 1 and NQO1. TSA treatment enhanced the expression of HO-1 and NQO1 in MIA mice (Fig. 4a). TSA treatment also suppressed OA-induced MMP-1, MMP-3, and MMP-13 expression (Fig. 4b) and significantly reduced tumor necrosis factor (TNF)- $\alpha$ (Fig. 4c), IL-1 $\beta$ (Fig. 4d), and IL-6 (Fig. 4e) levels. In addition, TSA treatment caused enhanced Nrf2 and histone 3 acetylation in mouse joints (Fig. 4f). These results demonstrate that HDACiimproved cartilage histology is associated with $\mathrm{HDACi}$ enhancement of tissue-protective molecules and repression of MMPs and proinflammatory cytokines. TSA protection of OA was correlated with its HDAC inhibition property, as it increased Nrf2 and histone 3 acetylation.

\section{TSA protection of OA requires Nrf2}

We next decided to determine whether Nrf2 was required for the HDACi protection. We injected MIA into both WT and Nrf2-KO mice and treated mice with TSA as before. In WT mice, TSA significantly reduced cartilage damage, including hypocellularity and loss of proteoglycans; however, TSA failed to reduce the joint damage in Nrf2-KO mice (Fig. 5). Similarly to previous results, WT mice had summed scores of $11.00 \pm 3.65$ for the femur and $13.50 \pm$ 5.23 for the tibia, whereas TSA treatment led to a significant improvement of $5.33 \pm 2.19$ for the femur and $6.92 \pm$ 4.24 for the tibia $(p<0.05)$ (Fig. $4 \mathrm{~b})$. When the maximum scores were applied, WT mice scored $2.17 \pm 0.80$ for the femur and $2.67 \pm 0.99$ for the tibia, whereas TSA showed significantly lower scores of $1.00 \pm 0.58$ for the femur and $1.25 \pm 0.63$ for the tibia $(p<0.05)$ (Fig. 4c). However, in Nrf2-KO mice, TSA treatment led to only a marginal reduction of joint cartilage damage (Fig. 5d). Nrf2-KO mice 


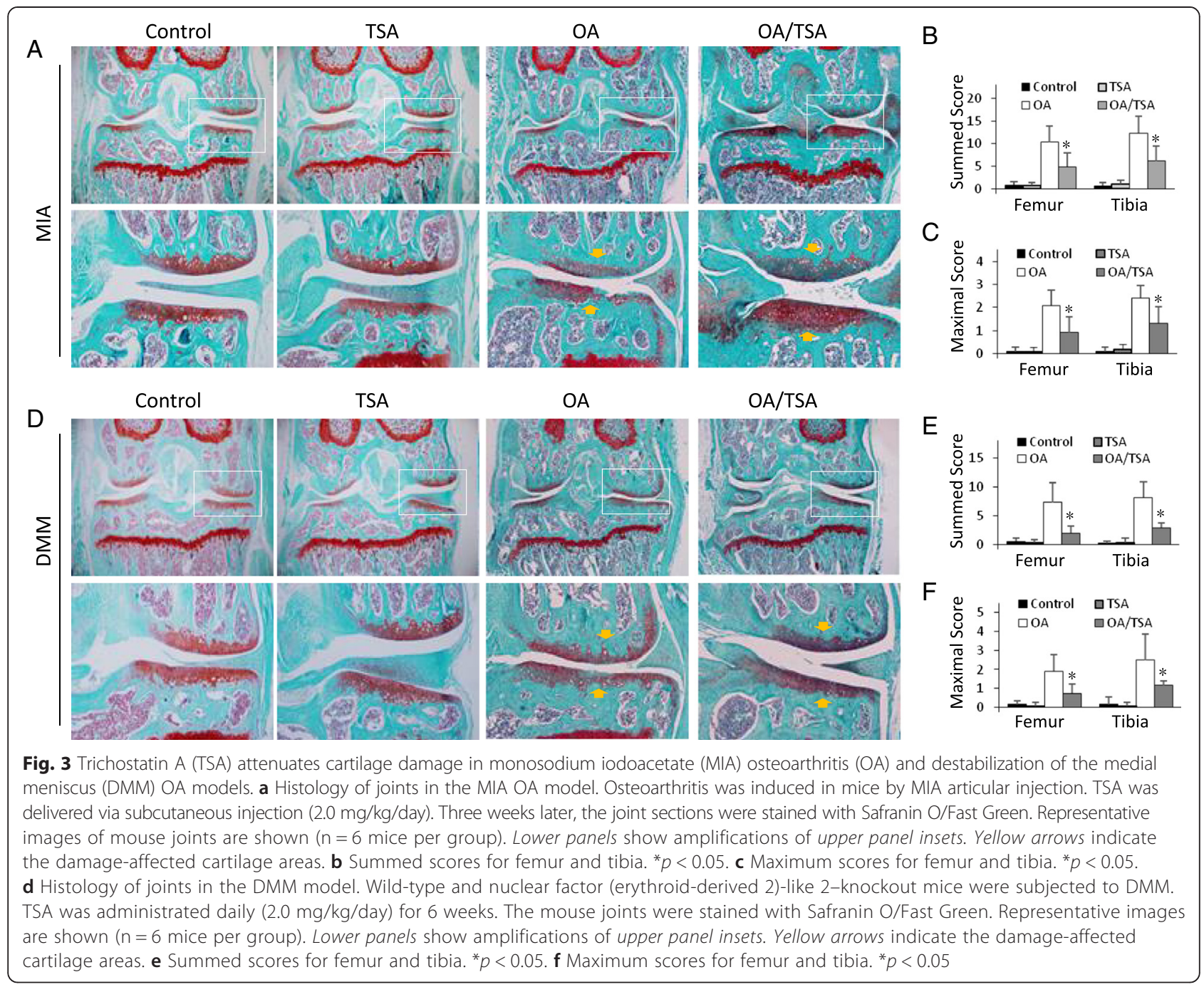

showed summed scores of $18.17 \pm 7.20$ for the femur and $20.42 \pm 8.65$ for the tibia, but TSA treatment only slightly reduced the scores to $14.17 \pm 6.39$ for the femur and $17.33 \pm 10.73$ for the tibia (Fig. 5b). Nrf2-KO mice had maximum scores of $3.50 \pm 1.41$ for the femur and $3.83 \pm 1.57$ for the tibia. TSA treatment was associated with similar scores of $3.25 \pm 1.87$ for the femur and $3.33 \pm 1.57$ for the tibia (Fig. 5c), indicating that TSA provided no protection against cartilage damage of OA in mice that lacked Nrf2. These results strongly indicate that TSA protects against cartilage damage in OA through regulation of Nrf2.

We observed similar histological changes in the DMM model (Fig. 5d). Among WT mice, the OA group had summed scores of $7.67 \pm 3.35$ for the femur and $9.08 \pm$ 3.22 for the tibia, whereas TSA treatment exhibited significantly lower values of $3.00 \pm 2.31$ and $3.33 \pm 0.94$, respectively $(p<0.05$ and $p<0.01)$ (Fig. 5e). The OA group had maximum scores of $2.17 \pm 0.90$ for the femur and $2.67 \pm 1.18$ for the tibia, whereas the TSA-treated group had significantly lower values of $0.92 \pm 0.79$ and $1.25 \pm 0.48$, respectively $(p<0.05)$ (Fig. $5 f)$. In contrast to TSA alleviation of cartilage destruction in WT mice, TSA treatment neither improved cartilage degradation nor changed the scores significantly in Nrf2-KO mice (Fig. 5e, f, right panels).

We also tested the MMP-1, MMP-3, MMP-13, HO-1, and NQO1 mRNA levels in cartilage by qRT-PCR. The results showed that TSA treatment significantly suppressed the induction of MMP-1, MMP-3, and MMP-13 (Fig. 5g) and enhanced the expression of HO-1 (Fig. 5h) and NQO1 (Fig. 5i) in WT mice, but it did not significantly change these levels in Nrf2-KO mice. Consistent with the histopathological changes, Nrf2-KO mice displayed significantly higher levels of MMP-1, MMP-3, and MMP-13 than WT mice, highlighting these MMPs' destructive role in OA.

\section{Discussion}

HDACi have been used in various animal models of arthritis and are effective in protection against arthritic 


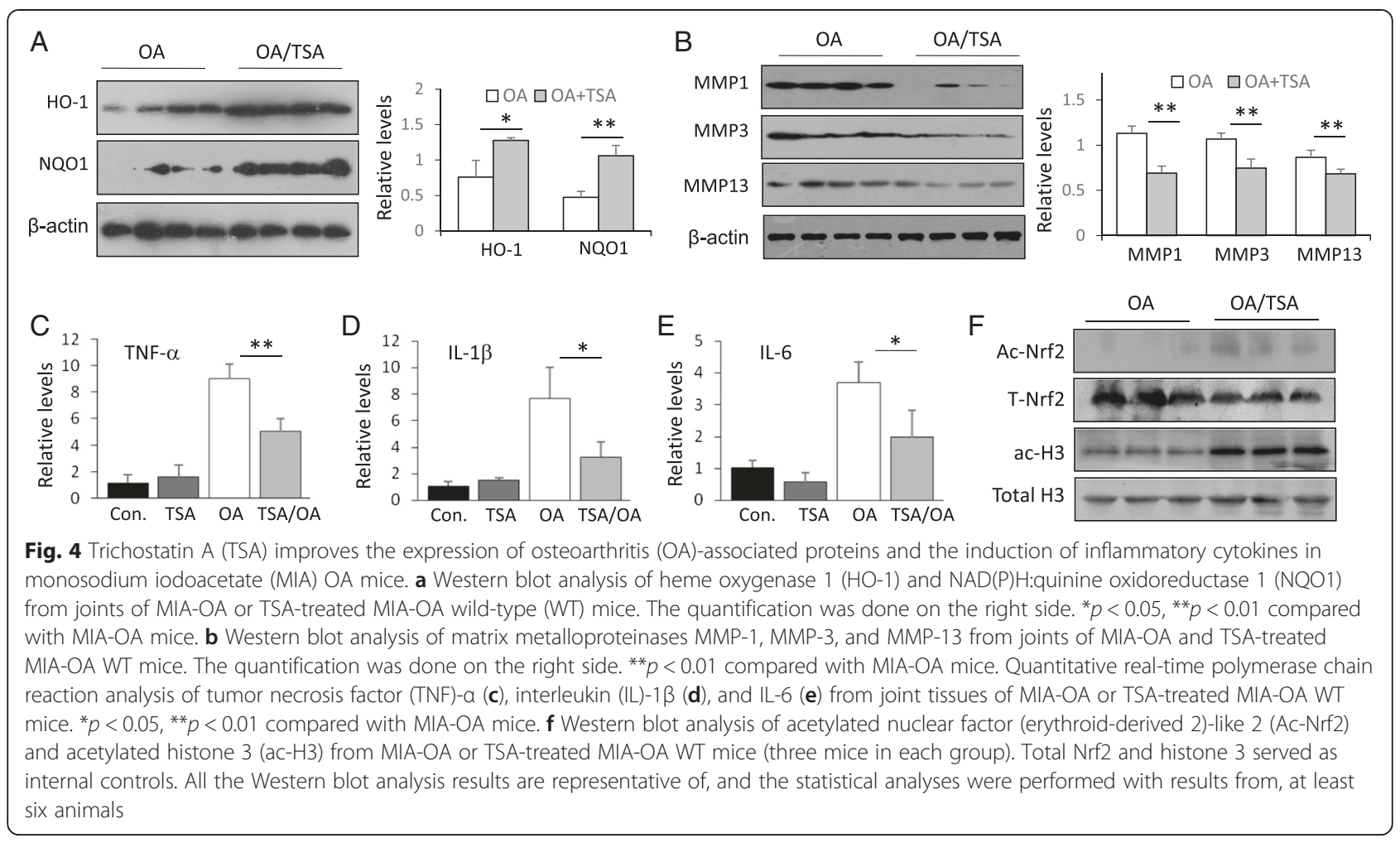

pathogenesis. Although many contributing factors and/ or molecules are proposed to account for the protective mechanisms of HDACi, we found in this study that Nrf2 acts upstream of the signaling and plays an indispensable role in HDACi-mediated protection against OA.

$\mathrm{OA}$ is one of the most common aging-related joint diseases, and the molecular mechanisms of OA are not yet fully known [21]. Excessive oxidative stress is associated with OA and triggers the programmed cell death or necrosis of chondrocytes during OA [22]. Proinflammatory cytokines such as TNF- $\alpha$, IL-1 $\beta$, IL-6, and MMPs also play major roles in OA pathogenesis [23, 24]. Nrf2 transcription factor is a master regulator of antioxidative stress. Nrf2 signaling regulates the expression of antioxidantresponsive genes and phase II detoxifying enzymes that counteract the oxidative damage in tissue injury [4]. The Nrf2 pathway also crosstalks with inflammatory signaling pathways such as the MAPK and nuclear factor $\mathrm{\kappa B}$ pathways and negatively regulates inflammatory responses $[25,26]$. Therefore, Nrf2 is expected to provide protection against OA injury. Previous studies demonstrated that Nrf2 was activated in the joints of mice and patients with rheumatoid arthritis and that depletion of Nrf2 accelerated joint inflammation and cartilage destruction in animals with rheumatoid arthritis [27, 28]. Although rheumatoid arthritis and OA share many common features of joint damage, they represent different etiological processes. We employed two OA animal models-namely, DMM and MIA - to verify the critical role of Nrf2 in OA development and progression. The DMM model is one of the most commonly used surgical OA models. In the DMM model, the traumatic injury causes a progressive degeneration of cartilage, subchondral bone sclerosis, and osteophyte formation, which mimic the pathological changes observed in human OA $[29,30]$. The MIA model represents the chemical induction of OA by intra-articular injection of MIA. MIA inhibits glyceraldehyde 3-phosphate dehydrogenase activity in chondrocytes and results in cell death following the disruption of the cellular glycolysis process [31]. The cartilage degenerative changes in MIA are comparable to those of human OA, including structural changes such as lack of surface regularities, cartilaginous matrix collapse, and cartilage fibrillation as well as functional changes such as reduction in the compressive stiffness of the articular cartilage [32]. We demonstrated in both DMM and MIA models that Nrf2 downstream proteins HO-1 and NQO1 were upregulated in OA. More importantly, mice lacking Nrf2 developed much more severe joint damage, as judged by histological examination and the summed and maximum score analyses (Fig. 1a-d). These results indicate that, similarly to the mouse model of rheumatoid arthritis, the Nrf2 pathway exerts a major protective function in OA development and pathogenesis.

Recent studies have revealed that Nrf2 is a protein acetylation target. Nrf2 acetylation increases its signaling and enhances its downstream gene expression $[7,8]$. We previously showed that HDACi induced Nrf2 acetylation and alleviated the stroke-related brain inflammatory 


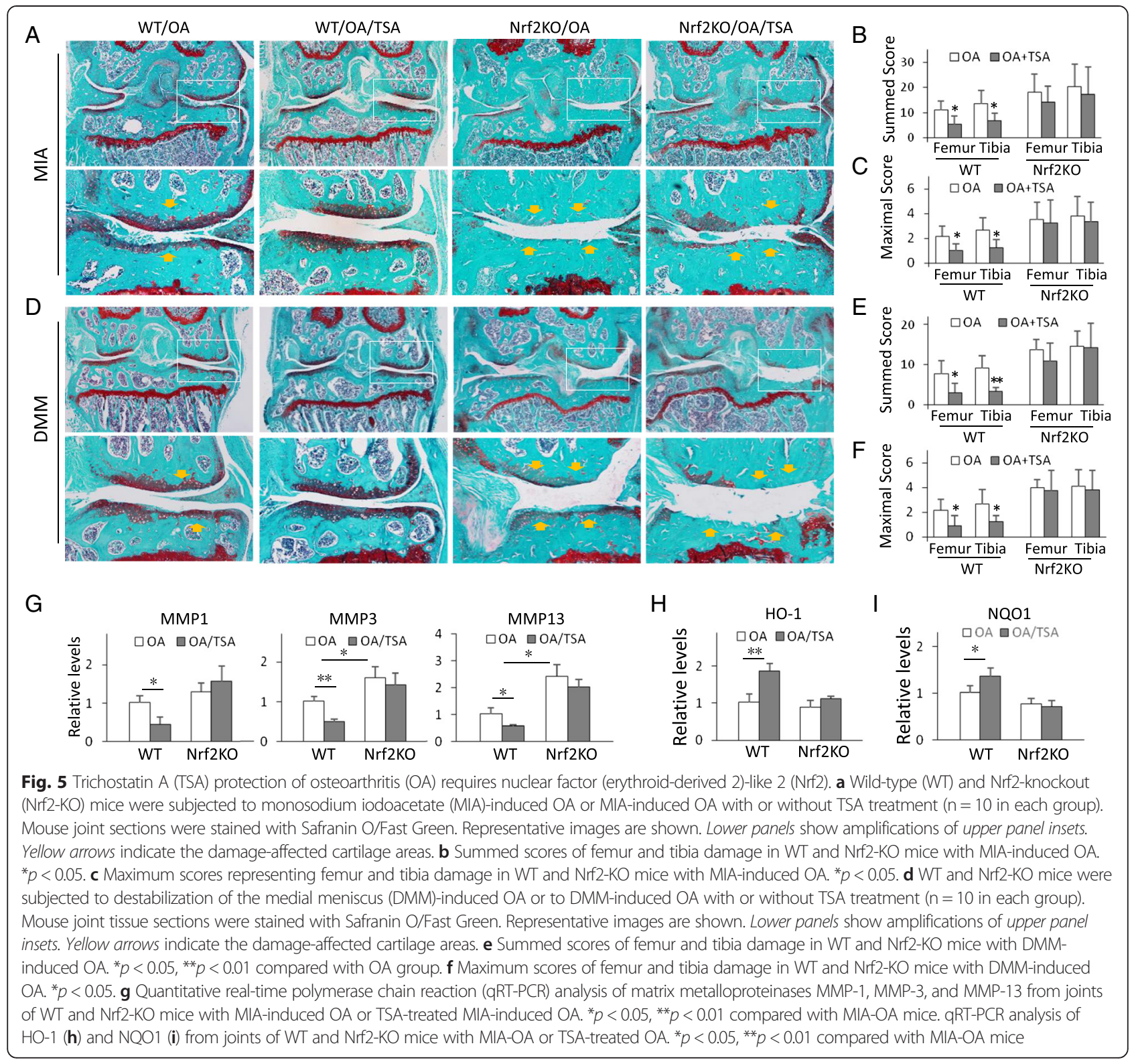

damage [20]. In the present study, we confirmed that the HDACi TSA promoted Nrf2 nuclear translocation, increased expression of Nrf2 target genes HO-1 and NQO1, and activated HO-1 promoter transactivation in chondrocytes (Fig. 2a-d), suggesting that TSA-induced Nrf2 acetylation might contribute to HDACi protection in OA. In the following experiments, we confirmed that TSA enhanced expression of Nrf2 downstream proteins HO-1 and NQO1 in mouse joints and effectively reduced cartilage damage in both MIA- and DMM-treated mice. Cartilage degradation is a hallmark of OA and is caused partially by the excess deposition of cartilage proteoglycan-degrading enzymes such as MMPs [33-35]. Proinflammatory cytokines also promote cartilage injuries [36]. Researchers in previous studies have reported that HDACi repressed MMPs and proinflammatory cytokine expression in joints and reduced the severity of cartilage lesions [13, 14, 37]. We also found that TSA effectively reduced the levels of MMP-1, MMP-3, MMP-13, TNF- $\alpha$, IL-1 $\beta$, and IL-6 in mouse joints (Fig. 3). In light of the fact that upregulation of Nrf2 downstream proteins such as HO-1 can reduce the expression of MMPs and inhibit the production of proinflammatory cytokines [38-40], it is reasonable to attribute the inhibition of MMPs and proinflammatory cytokines, at least partially, to the HDACi-induced Nrf2 signaling activation. Also, because HO-1 and the other Nrf2 target proteins are major defenders in tissue repair [41], we speculate that HDACiinduced Nrf2 activation is the major contributor to the improved joint histology. However, it is worth noting that the mice used in our experiments were relatively young 
(around 8 weeks old) and had immature skeletons. We therefore cannot exclude the possibility that other late developmental events might affect the effectiveness of HDACi and Nrf2 dependency in these two OA models. Future study is needed to examine whether HDACi has similar protective effects in older mice.

To conclusively determine the role of Nrf2 acetylation in HDACi protection against OA, we further compared the effect of TSA on cartilage destruction between WT and Nrf2-KO mice. Our results showed that, although TSA still markedly reduced OA cartilage damage in WT mice, it did not significantly improve the joint injury in Nrf2-KO mice in either the MIA or the DMM model (Fig. 5), indicating that Nrf2 is essential for HDACimediated cartilage protection. Previous studies have shown that various types of pan-HDACi and specific HDAC subtype inhibition provide protection against $\mathrm{OA}$ injuries [13-16]; however, the acetylation target molecules and the signaling pathways involved are largely unknown. HDACs can either modify non-histone signal molecules, such as Nrf2 in the present study, or target chromatin histones independently to affect gene transcription. HDAC inhibition can also promote signaling molecule association with HAT, bringing HAT to the target gene promoter and subsequently affecting target gene transcription [42]. Our results show that Nrf2-KO mice are not protected by TSA (Fig. 5), suggesting that Nrf2 represents a convergence between Nrf2 acetylation and HDACi actions through which HDAC inhibition provides protection against $\mathrm{OA}$.

\section{Conclusions}

We explored the effectiveness of HDACi in protection against OA in two animal models and identified Nrf2 as the critical molecule mediating HDACi protection. Our study improves understanding of OA pathogenesis and provides novel insights into the development of HDACibased therapeutic strategies.

\footnotetext{
Abbreviations

ac-H3: acetylated histone 3; ARE: antioxidant response element; DMM: destabilization of the medial meniscus; GAPDH: glyceraldehyde 3-phosphate dehydrogenase; HAT: histone acetyltransferase; HDAC: histone deacetylase; HDACi: histone deacetylase inhibitor; HO-1: heme oxygenase 1; IACUC: Institutional Animal Care and Use Committee; IL: interleukin; KO: knockout; MAPK: mitogen-activated protein kinase; MIA: monosodium iodoacetate; MM: medial meniscus; MMP: matrix metalloproteinase; MMTL: medial meniscotibial ligament; NQO1: NAD(P)H:quinine oxidoreductase 1; Nrf2: nuclear factor (erythroid-derived 2)-like 2; Nrf2-KO: Nrf2-knockout; OA: osteoarthritis; OARSI: Osteoarthritis Research Society International; PBS: phosphate-buffered saline; qRT-PCR: quantitative real-time polymerase chain reaction; RT-PCR: reverse transcriptase polymerase chain reaction; TNF-a: tumor necrosis factor alpha; TSA: trichostatin A; WT: wild type.
}

\section{Competing interests}

The authors declare that they have no competing interests.

\section{Authors' contributions}

DC and SY performed the most experiments, collected and analyzed data, drafted the initial manuscript, and participated in revising the manuscript. JY participated in Western blot, RT-PCR, and animal experiments, performed the statistical analysis, and helped to revise the manuscript. QJ participated in study conception and design and revised all manuscript drafts critically for important intellectual content. WC was responsible for study conception and design, acquisition of data, analysis and interpretation of data, and drafting of the manuscript and revising it critically for important intellectual content. All authors read and approved the final manuscript.

\section{Acknowledgments}

This work was supported by research grants from the National Science Fund for Distinguished Young Scholars of China (grant 81125013) and the National Natural Science Foundation of China (grants 81271301 and 81470940). We thank Siyuan Qin for help with mouse work.

\section{Author details}

${ }^{1}$ Jiangsu Key Laboratory of Molecular Medicine, Nanjing University School of Medicine, Nanjing 210093, People's Republic of China. ${ }^{2}$ Center of Diagnosis and Treatment for Joint Disease, Nanjing Drum Tower Hospital Affiliated with Nanjing University School of Medicine, Nanjing 210008, People's Republic of China. ${ }^{3}$ Model Animal Research Center of Nanjing University, Nanjing 210032, People's Republic of China. ${ }^{4}$ National Clinical Research Center of Kidney Diseases, Jinling Hospital, Nanjing University School of Medicine, Nanjing 210016, People's Republic of China.

Received: 3 February 2015 Accepted: 4 September 2015

Published online: 26 September 2015

\section{References}

1. Bijlsma JW, Berenbaum F, Lafeber FP. Osteoarthritis: an update with relevance for clinical practice. Lancet. 2011;377:2115-26. doi:10.1016/ S0140-6736(11)60243-2.

2. Sellam J, Berenbaum F. Is osteoarthritis a metabolic disease? Joint Bone Spine. 2013;80:568-73. doi:10.1016/j.jbspin.2013.09.007.

3. Su SC, Tanimoto K, Tanne Y, Kunimatsu R, Hirose N, Mitsuyoshi T, et al. Celecoxib exerts protective effects on extracellular matrix metabolism of mandibular condylar chondrocytes under excessive mechanical stress. Osteoarthritis Cartilage. 2014;22:845-51. doi:10.1016/j.joca.2014.03.011.

4. de Vries HE, Witte M, Hondius D, Rozemuller AJ, Drukarch B, Hoozemans J, et al. Nrf2-induced antioxidant protection: a promising target to counteract ROS-mediated damage in neurodegenerative disease? Free Radic Biol Med. 2008;45:1375-83. doi:10.1016/j.freeradbiomed.2008.09.001.

5. Zhao J, Zhang B, Li S, Zeng L, Chen Y, Fang J. Mangiferin increases Nrf2 protein stability by inhibiting its ubiquitination and degradation in human HL60 myeloid leukemia cells. Int J Mol Med. 2014;33:1348-54. doi:10.3892/ijmm.2014.1696.

6. Apopa PL, He X, Ma Q. Phosphorylation of Nrf2 in the transcription activation domain by casein kinase 2 (CK2) is critical for the nuclear translocation and transcription activation function of Nrf2 in IMR-32 neuroblastoma cells. J Biochem Mol Toxicol. 2008;22:63-76. doi:10.1002/jbt.20212.

7. Sun Z, Chin YE, Zhang DD. Acetylation of Nrf2 by p300/CBP augments promoter-specific DNA binding of Nrf2 during the antioxidant response. Mol Cell Biol. 2009;29:2658-72. doi:10.1128/MCB.01639-08.

8. Kawai Y, Garduno L, Theodore M, Yang J, Arinze IJ. Acetylation-deacetylation of the transcription factor Nrf2 (nuclear factor erythroid 2-related factor 2) regulates its transcriptional activity and nucleocytoplasmic localization. J Biol Chem. 2011;286:7629-40. doi:10.1074/jbc.M110.208173.

9. Patra N, De U, Kim TH, Lee YJ, Ahn MY, Kim ND, et al. A novel histone deacetylase (HDAC) inhibitor MHY219 induces apoptosis via up-regulation of androgen receptor expression in human prostate cancer cells. Biomed Pharmacother. 2013;67:407-15. doi:10.1016/j.biopha.2013.01.006.

10. Hansen FK, Sumanadasa SD, Stenzel K, Duffy S, Meister S, Marek L, et al. Discovery of HDAC inhibitors with potent activity against multiple malaria parasite life cycle stages. Eur J Med Chem. 2014;82:204-13. doi:10.1016/ j.ejmech.2014.05.050.

11. Foti SB, Chou A, Moll AD, Roskams AJ. HDAC inhibitors dysregulate neural stem cell activity in the postnatal mouse brain. Int J Dev Neurosci. 2013;31:434-47. doi:10.1016/j.ijdevneu.2013.03.008.

12. Herold C, Ganslmayer M, Ocker M, Hermann M, Geerts A, Hahn EG, et al. The histone-deacetylase inhibitor trichostatin A blocks proliferation and triggers apoptotic programs in hepatoma cells. J Hepatol. 2002;36:233-40. 
13. Culley KL, Hui W, Barter MJ, Davidson RK, Swingler TE, Destrument AP, et al. Class I histone deacetylase inhibition modulates metalloproteinase expression and blocks cytokine-induced cartilage degradation. Arthritis Rheum. 2013;65:1822-30. doi:10.1002/art.37965.

14. Chen WP, Bao JP, Hu PF, Feng J, Wu LD. Alleviation of osteoarthritis by trichostatin A, a histone deacetylase inhibitor, in experimental osteoarthritis. Mol Biol Rep. 2010;37:3967-72. doi:10.1007/s11033-010-0055-9.

15. Li M, Liu X, Sun X, Wang Z, Guo W, Hu F, et al. Therapeutic effects of NK-HDAC-1, a novel histone deacetylase inhibitor, on collagen-induced arthritis through the induction of apoptosis of fibroblast-like synoviocytes. Inflammation. 2013;36:888-96. doi:10.1007/s10753-013-9616-0.

16. Joosten LA, Leoni F, Meghii S, Mascagni P. Inhibition of HDAC activity by ITF2357 ameliorates joint inflammation and prevents cartilage and bone destruction in experimental arthritis. Mol Med. 2011;17:391-6. doi:10.2119/ molmed.2011.00058

17. Ogbonna AC, Clark AK, Gentry C, Hobbs C, Malcangio M. Pain-like behaviour and spinal changes in the monosodium iodoacetate model of osteoarthritis in C57Bl/6 mice. Eur J Pain. 2013;17:514-26. doi:10.1002/j.1532-2149.2012.00223.x.

18. Glasson SS, Askew R, Sheppard B, Carito BA, Blanchet T, Ma HL, et al. Characterization of and osteoarthritis susceptibility in ADAMTS-4-knockout mice. Arthritis Rheum. 2004;50:2547-58. doi:10.1002/art.20558.

19. La Porta C, Bura SA, Aracil-Fernández A, Manzanares J, Maldonado R. Role of CB1 and CB2 cannabinoid receptors in the development of joint pain induced by monosodium iodoacetate. Pain. 2013;154:160-74. doi:10.1016/ j.pain.2012.10.009.

20. Wang B, Zhu X, Kim Y, Li J, Huang S, Saleem S, et al. Histone deacetylase inhibition activates transcription factor Nrf2 and protects against cerebral ischemic damage. Free Radic Biol Med. 2012;52:928-36. doi:10.1016/ j.freeradbiomed.2011.12.006.

21. Kawaguchi H. Mechanism of molecular backgrounds of osteoarthritis. Nihon Rinsho. 2014;72:1729-33. Japanese.

22. Jiang L, Li L, Geng C, Gong D, Jiang L, Ishikawa N, et al. Monosodium iodoacetate induces apoptosis via the mitochondrial pathway involving ROS production and caspase activation in rat chondrocytes in vitro. J Orthop Res. 2013;31:364-9. doi:10.1002/jor.22250.

23. Furuzawa-Carballeda J, Macip-Rodríguez PM, Cabral AR. Osteoarthritis and rheumatoid arthritis pannus have similar qualitative metabolic characteristics and pro-inflammatory cytokine response. Clin Exp Rheumatol. 2008;26:554-60.

24. Chorazy-Massalska M, Kontny E, Kornatka A, Rell-Bakalarska M, Marcinkiewicz J, Maslinski W. The effect of taurine chloramine on pro-inflammatory cytokine production by peripheral blood mononuclear cells isolated from rheumatoid arthritis and osteoarthritis patients. Clin Exp Rheumatol. 2004:22:692-8.

25. Anuranjani, Bala M. Concerted action of Nrf2-ARE pathway, MRN complex, HMGB1 and inflammatory cytokines - implication in modification of radiation damage. Redox Biol. 2014;2:832-46. doi:10.1016/j.redox.2014.02.008.

26. So H, Kim H, Kim Y, Kim E, Pae HO, Chung HT, et al. Evidence that cisplatin-induced auditory damage is attenuated by downregulation of pro-inflammatory cytokines via Nrf2/HO-1. J Assoc Res Otolaryngol. 2008:9:290-306. doi:10.1007/s10162-008-0126-y.

27. Wruck CJ, Fragoulis A, Gurzynski A, Brandenburg LO, Kan YW, Chan K, et al. Role of oxidative stress in rheumatoid arthritis: insights from the Nrf2-knockout mice. Ann Rheum Dis. 2011;70:844-50. doi:10.1136/ ard.2010.132720

28. Maicas N, Ferrándiz ML, Brines R, Ibáñez L, Cuadrado A, Koenders MI, et al. Deficiency of Nrf2 accelerates the effector phase of arthritis and aggravates joint disease. Antioxid Redox Signal. 2011;15:889-901. doi:10.1089/ars.2010.3835.

29. Hashimoto S, Rai MF, Janiszak KL, Cheverud JM, Sandell LJ. Cartilage and bone changes during development of post-traumatic osteoarthritis in selected LGXSM recombinant inbred mice. Osteoarthritis Cartilage. 2012;20:562-71. doi:10.1016/j.joca.2012.01.022.

30. Little CB, Zaki S. What constitutes an "animal model of osteoarthritis" - the need for consensus? Osteoarthritis Cartilage. 2012;20:261-7. doi:10.1016/ j.joca.2012.01.017.

31. van der Kraan PM, Vitters EL, van de Putte LB, van den Berg WB. Development of osteoarthritic lesions in mice by "metabolic" and "mechanical" alterations in the knee joints. Am J Pathol. 1989;135:1001-14.
32. Naveen SV, Ahmad RE, Hui WJ, Suhaeb AM, Murali MR, Shanmugam R, et al. Histology, glycosaminoglycan level and cartilage stiffness in monoiodoacetate-induced osteoarthritis: comparative analysis with anterior cruciate ligament transection in rat model and human osteoarthritis. Int J Med Sci. 2014;11:97-105. doi:10.7150/ijms.6964.

33. Kaspiris A, Khaldi L, Grivas TB, Vasiliadis E, Kouvaras I, Dagkas S, et al. Subchondral cyst development and MMP-1 expression during progression of osteoarthritis: an immunohistochemical study. Orthop Traumatol Surg Res. 2013;99:523-9. doi:10.1016/j.otsr.2013.03.019.

34. Ryu JH, Lee A, Huh MS, Chu J, Kim K, Kim BS, et al. Measurement of MMP activity in synovial fluid in cases of osteoarthritis and acute inflammatory conditions of the knee joints using a fluorogenic peptide probe-immobilized diagnostic kit. Theranostics. 2012;2:198-206. doi:10.7150/thno.3477.

35. Lee YJ, Lee EB, Kwon YE, Lee JJ, Cho WS, Kim HA, et al. Effect of estrogen on the expression of matrix metalloproteinase (MMP)-1, MMP-3, and MMP-13 and tissue inhibitor of metalloproternase- 1 in osteoarthritis chondrocytes. Rheumatol Int. 2003;23:282-8. doi:10.1007/s00296-003-0312-5.

36. Kapoor M, Martel-Pelletier J, Lajeunesse D, Pelletier JP, Fahmi H. Role of proinflammatory cytokines in the pathophysiology of osteoarthritis. Nat Rev Rheumatol. 2011;7:33-42. doi:10.1038/nrrheum.2010.196.

37. Nasu Y, Nishida K, Miyazawa S, Komiyama T, Kadota Y, Abe N, et al. Trichostatin A, a histone deacetylase inhibitor, suppresses synovial inflammation and subsequent cartilage destruction in a collagen antibody-induced arthritis mouse model. Osteoarthritis Cartilage. 2008;16:723-32. doi:10.1016/j.joca.2007.10.014.

38. Clérigues V, Murphy CL, Guillén MI, Alcaraz MJ. Haem oxygenase-1 induction reverses the actions of interleukin-1 $\beta$ on hypoxia-inducible transcription factors and human chondrocyte metabolism in hypoxia. Clin Sci (Lond). 2013;125:99-108. doi:10.1042/CS20120491.

39. Clérigues V, Guillén MI, Castejón MA, Gomar F, Mirabet V, Alcaraz MJ. Heme oxygenase-1 mediates protective effects on inflammatory, catabolic and senescence responses induced by interleukin-1 $\beta$ in osteoarthritic osteoblasts. Biochem Pharmacol. 2012;83:395-405. doi:10.1016/ j.bcp.2011.11.024.

40. Guillén M, Megías J, Gomar F, Alcaraz M. Haem oxygenase-1 regulates catabolic and anabolic processes in osteoarthritic chondrocytes. J Pathol. 2008;214:515-22. doi:10.1002/path.2313.

41. Wagener FA, Scharstuhl A, Tyrrell RM, Von den Hoff JW, Jozkowicz A, Dulak J, et al. The heme-heme oxygenase system in wound healing; implications for scar formation. Curr Drug Targets. 2010;11:1571-85.

42. Adcock IM, Lee KY. Abnormal histone acetylase and deacetylase expression and function in lung inflammation. Inflamm Res. 2006;55:311-21. doi:10.1007/s00011-006-0081-1.

\section{Submit your next manuscript to BioMed Central and take full advantage of:}

- Convenient online submission

- Thorough peer review

- No space constraints or color figure charges

- Immediate publication on acceptance

- Inclusion in PubMed, CAS, Scopus and Google Scholar

- Research which is freely available for redistribution 